\title{
Entrevista
}

\section{Alfabetización inicial desde una perspectiva constructivista psicogenética. Una entrevista con Delia Lerner y Mirta Castedo}

\author{
Marta Alegría \\ Universidad Nacional de la Plata y Universidad Alberto Hurtado \\ Tatiana Cisternas \\ Universidad Alberto Hurtado
}

(Texto recibido el 29 de junio de 2018; aceptado el 4 de julio de 2018)

DOI: http://doi.org/10.5565/rev/jt13.774

\begin{abstract}
Delia Lerner es Profesora Consulta de la Facultad de Filosofía y Letras e investigadora en Didáctica de la Lectura y la Escritura en el Instituto de Investigaciones en Ciencias de la Educación (IICE)-Universidad de Buenos Aires. Docente de la Maestría en Escritura y Alfabetización de la Universidad Nacional de La Plata. Asesora -y ha asesorado- programas de formación docente continua en Argentina y en otros países latinoamericanos. Es autora de diversas publicaciones en su área de especialidad.

Mirta Castedo es Profesora titular en Facultad de Humanidades y Ciencias de la Educación e investigadora en el Instituto de Investigaciones en Ciencias Sociales (IdICS), Universidad Nacional de La Plata. Creó y dirigió la Maestría en Escritura y Alfabetización entre 2006 y 2016. Coordinó y dirigió diversos programas de formación de docentes en distintos países de América Latina. Autora de publicaciones sobre enseñanza de la lectura y la escritura.
\end{abstract}

\section{Entrevista (Abril, 2018)}

MA (María Alegría): Delia Lerner, Mirta Castedo agradecemos mucho esta oportunidad de conversar con ustedes. En término generales, nos interesa que podamos reflexionar sobre algunas cuestiones centrales de sus propuestas didácticas, los fundamentos que le subyacen y los desafíos latinoamericanos para mejorar la enseña de la lectura y la escritura en nuestras aulas. Para empezar... ¿Cuáles han sido las principales preocupaciones de investigación en alfabetización inicial desde la perspectiva constructivista psicogenética?, ¿cómo caracterizarían esas investigaciones y cuál es el objeto de estudio?, ¿cómo nacieron y cuáles son los intereses actuales? 
DL (Delia Lerner): La preocupación original fue la de construir una propuesta didáctica que tomara en cuenta las conceptualizaciones infantiles acerca del sistema de escritura y contribuyera así a evitar, o al menos a disminuir, la repitencia en los primeros años. La cuestión del fracaso escolar, que más tarde Emilia Ferreiro caracterizaría como fracaso de la escuela en la alfabetización, estuvo en el origen de la investigación en las aulas, además de ser uno de los móviles que impulsaron la investigación psicogenética. Acercar la enseñanza al aprendizaje, suponíamos ya en esa época (alrededor de 1980) es crucial para que puedan avanzar muchos niños que se encuentran en situación de dificultad. Podríamos considerar que esta sigue siendo nuestra hipótesis nuclear. Elaboramos unas "líneas de trabajo" que incluían diferentes tipos de actividades de lectura y escritura y decidimos ponerlas en práctica para analizar cómo se desarrollaban en el aula, qué se podría aprender a través de ellas, cuáles podían ser productivas para que todos o algunos niños avanzaran, qué intervenciones del maestro parecían contribuir a esos avances. Como ignorábamos qué pasaría al trabajar con una propuesta de enseñanza que se apartaba fuertemente de los métodos tradicionales (fonéticos, en particular, "palabra generadora”) y ponía en primer plano las ideas y modos de aprendizaje de los niños, las interacciones cognitivas entre ellos y también su participación en la cultura escrita, teníamos que observar y analizar lo que sucedía en la clase con esa propuesta. Fue así como comenzamos a hacer investigación didáctica sin saberlo.

TC (Tatiana Cisternas): ¿Qué entendemos por investigación didáctica?

DL: Según la conceptualización original formulada en Didáctica de las Matemáticas por Guy Brousseau (cuyo trabajo comenzamos a conocer en 1987 gracias a Myriam Nemirovsky y que hoy es compartida por diferentes didácticas específicas), el objeto de estudio puede definirse así: las relaciones entre la enseñanza y el aprendizaje de determinados contenidos, propios de cierta área del conocimiento. El lugar donde es posible estudiar estas relaciones es el aula. Es por eso que, aunque se recurre a métodos auxiliares (entrevistas, análisis de diseños curriculares, de manuales, de programas informáticos, etc.), el método central es el análisis del desarrollo en las clases de propuestas de enseñanza. La investigación didáctica puede ser "naturalista", cuando estudia las relaciones entre enseñanza y aprendizaje tal como se dan en las aulas, sin que el investigador haya intervenido en la elaboración de la propuesta, o bien puede centrase en el estudio de proyectos de enseñanza elaborados por los investigadores o, cada vez más, producidos en conjunto por maestros e investigadores. Este último tipo de 
investigación es el que ha caracterizado en forma predominante nuestro trabajo en alfabetización inicial.

Los proyectos de enseñanza cuyo desarrollo en el aula aún seguimos estudiando, se caracterizan por incluir cuatro tipos fundamentales de situaciones: lectura y escritura a través del docente, lectura y escritura de los niños por sí mismos. Mucha investigación y diversas experiencias escolares sistematizadas fueron necesarias para diseñar y analizar registros de clase correspondientes a estas diferentes situaciones, para explicitar, por ejemplo, en qué condiciones didácticas las situaciones de lectura de los niños por sí mismos son favorables para producir avances en su aprendizaje como lectores, para que las interacciones cognitivas entre los niños resulten productivas $\mathrm{y}$, sobre todo, para ir afinando las intervenciones del docente que contribuyen a tender puentes entre el estado de conocimientos de los niños y lo que se apunta a enseñar.

MC (Mirta Castedo): Como señalaba Delia, en general existe cierto consenso en estos dos tipos de investigaciones. Por una parte, las investigaciones que también son llamadas de intervención o "experimentales" pero no en el sentido de que exista un grupo control y uno experimental sino en que se hace una "experimentación" en el aula para estudiar cómo se desarrolla un proyecto de enseñanza (en sentido amplio, puede ser una secuencia de clases). Este tipo de investigación se realiza cuando el objeto de enseñanza no existe aún en las aulas o cuando existe pero se quiere analizar un modo diferente de enseñarlo. A diferencia de la experimentación clásica, no se procede por comparación entre grupo experimental y grupo control sino por contrastación entre hipótesis a priori de la puesta en aula -las hipótesis didácticas- y su comprobación, refutación o ajuste a posteriori de la acción. Para llegar a construir esas hipótesis didácticas iniciales se realizan muchos análisis antes de la puesta en aula. Siempre se parte del análisis del campo de restricciones sobre el cual se va a desarrollar la acción didáctica: qué tipo de escuela, su historia institucional, su historia de prácticas, su proyecto de enseñanza más global, etc. porque las diferentes ideas que impregnan las prácticas ya instituidas hacen que un nuevo proyecto de enseñanza encarne de maneras muy diferentes y esto no es un dato anecdótico sino esencial en el resultado de la investigación. Toda escuela tiene un proyecto de enseñanza o varios en tensión, implícitos o explícitos, y acuerdos sobre su proyecto formativo. Tratar de entender en qué campo se va a insertar el nuevo proyecto para un o unos contenidos acotados es fundamental. Otro análisis preliminar es el epistemológico del contenido de aprendizaje (por ejemplo, qué se entiende por escritura 
o por revisión de textos en las prácticas sociales y en distintas disciplinas de referencia). Como casi siempre hay distintas maneras de concebir el contenido, es indispensable pararse en una manera de entenderlo para después poder comprender de qué modo se modifica con la enseñanza y en el aprendizaje. Otro análisis fundamental para nosotros es el de las concepciones e ideas de los alumnos sobre aquello que se va a enseñar, las dificultades y obstáculos que van a encontrar para asimilarlo. Aquí la referencia a estudios psicolingüísticos es fundamental pero a veces no alcanzan. En esos casos se realizan entrevistas o se desarrollan ciertas situaciones con algunos niños "en el margen" del aula o se analizan producciones o datos obtenidos de experiencias previas que no fueron planteadas como investigación. Esto se hace para tener una anticipación de respuestas de los chicos que permita diseñar intervenciones posibles para hacerlos avanzar. Por último, tenemos el análisis de la enseñanza "usual" de ese o esos contenidos y de sus consecuencias no solo en la escuela que se va a estudiar sino en general. La idea es que la nueva puesta en aula se instala en prácticas anteriores que van a filtrar sus efectos a través de contratos ya aprendidos. En ese sentido, el diseño del proyecto de enseñanza tiene muy en cuenta cómo evitar consecuencias no deseadas, que alterarían el sentido del proyecto.

TC: ¿La producción de conocimiento sobre la alfabetización se realiza solamente desde la investigación didáctica?

DL: Si bien la investigación didáctica es la fuente más importante de conocimiento acerca de la enseñanza y el aprendizaje de la lectura y la escritura, está lejos de ser la única. Creo que hemos producido conocimiento didáctico a través de experiencias escolares documentadas, a través de instancias de desarrollo curricular -que nos permitían estudiar lo que sucedía en algunas aulas al trabajar con situaciones didácticas que habíamos propuesto en documentos preparatorios del Diseño ${ }^{\mathrm{i}}$ - e incluso en el proceso de elaboración de los diseños curriculares. Cuando comenzamos a trabajar en diseño curricular -en la Secretaría de Educación de la Ciudad de Buenos Aires, al principio de la década de los 90-, enfrentamos un desafío: si bien habíamos escrito artículos o libros en los que analizábamos el trabajo realizado en alfabetización inicial en diferentes países latinoamericanos, por primera vez nos veíamos obligadas a sistematizar lo que sabíamos hasta ese momento acerca de las situaciones didácticas y las intervenciones docentes durante su transcurso. Por otra parte, dado que formular contenidos es central cuando se diseña un curriculo, fue también la primera vez que 
tuvimos que explicitar cómo concebíamos el objeto de enseñanza y desplegar los contenidos que aspirábamos a enseñar.

En relación con las situaciones didácticas, fue este desafío el que nos llevó a analizar situaciones muy diversas que habíamos propuesto y estudiado en las aulas y a agruparlas en los cuatro grandes tipos antes mencionados (situaciones de lectura y situaciones de escritura a través del docente; situaciones de lectura y situaciones de escritura de los niños por sí mismos). Por supuesto, cada tipo de situaciones incluye una diversidad marcada por el género que se lee o escribe, por el propósito de la lectura o la escritura.... Esta sistematización permitió plantear la posibilidad de que cada proyecto de enseñanza articulara los cuatro tipos de situaciones, lo que permitía trabajar simultáneamente sobre la adquisición del sistema de escritura y del lenguaje escrito, así como sobre diferentes facetas de las prácticas de lectura y escritura.

Por otra parte, fue el trabajo curricular el que nos llevó a tomar plena conciencia de que nuestro proyecto de enseñanza no involucraba sólo un cambio metodológico sino también un cambio fuerte en el objeto de enseñanza. Definir el objeto de enseñanza como "Prácticas del Lenguaje" fue una manera de plasmar nuestra intención de poner en primer plano en la escuela la lectura y la escritura en tanto prácticas sociales, de subrayar que el propósito de formar lectores y escritores requiere que se dedique gran parte del tiempo a leer y a escribir (más que a "estudiar Lengua"). Esta definición fue posible gracias a los aportes de diferentes ciencias que estudian la lectura y la escritura: no sólo de la Psicolingüística sino también de la Historia de la Lectura (Roger Chartier, Jean Hébrard, Guguielmo Cavallo) y luego de la Sociología de la Lectura y de la Escritura (Bernard Lahire). Desplegar los contenidos en términos de quehaceres del lector y quehaceres del escritor hubiera sido imposible sin recurrir a estos aportes. Son contenidos que indudablemente hoy habría que revisar en función de los avances de las diferentes ciencias que estudian la lectura y la escritura.

Por supuesto, antes de que iniciáramos el trabajo curricular, ya estaba claro -gracias a los aportes de Emilia Ferreiro y sus colaboradoras- que enseñar a leer y a escribir es algo muy diferente de enseñar una técnica, que el sistema de escritura es un objeto de conocimiento para los sujetos, que estos se plantean diversos problemas cognitivos y elaboran conceptualizaciones originales al intentar apropiarse de él. También estaba claro -ya lo dijimos- que acercar la enseñanza al aprendizaje suponía tomar en consideración a los sujetos en tanto productores de conocimiento sobre el sistema de escritura. Sin embargo, al formular los contenidos, no fue fácil circunscribir los aspectos que podían considerarse como objeto de 
enseñanza y aprendizaje escolar, distinguiéndolos de aquellos que son parte del proceso de reconstrucción realizado por el sujeto del aprendizaje.

MA: ¿Hay otras investigaciones, además de las didácticas, que se realizan en las aulas y que pueden aportar conocimiento acerca de la lectura y la escritura?

MC: Si bien la discusión acerca de qué es y qué no es investigación en didáctica específica es reciente y el estado actual es controvertido e inclusive, en ocasiones tiene límites difusos, está claro que varias disciplinas pueden estudiar el aula donde se está enseñando a leer y a escribir: el Análisis del Discurso, la Etnografía, la Sociología, la Historia, la Psicolingüística, etc. El solo hecho de estudiar el aula no los hace estudios didácticos. Es necesario que se ocupen de la relación entre enseñanza y aprendizaje $y$, en nuestra perspectiva, que asuman que enseñanza no es igual a aprendizaje y que el aprendizaje es un proceso de aproximaciones sucesivas porque existe la reconstrucción de los objetos de enseñanza por parte de los niños.

MA: ¿Eso supone que ustedes no toman en cuenta esos otros estudios?

MC: En absoluto, todo lo contrario. Para nosotros, además de la investigación de Emilia Ferreiro, los trabajos de Elsie Rockwell, de Judith Kalman, de Bernard Lahire, de Anne-Marie Chartier, entre otros, son una referencia fundamental para seguir comprendiendo por qué sucede lo que sucede en el aula y para seguir construyendo propuestas cada vez más viables y precisas que ayuden a todos los chicos a aprender a leer y a escribir. Pero esas propuestas hay que ponerlas a prueba en las aulas, esa es la especificidad de la investigación didáctica.

TC: Es frecuente encontrar trabajos que consideran posible deducir de teorías lingüísticas o psicológicas consecuencias para la enseñanza, para la alfabetización inicial en la escuela. ¿Qué las distingue de esas posturas?

DL: Creo que lo que nos distingue de las posturas "aplicacionistas" es que estamos convencidos de que lo didáctico es tan complejo que requiere ser estudiado en sí mismo. Ni la Psicología ni la Lingüística -que son indudablemente ciencias de referencia para la Didáctica de la lectura y la escritura- estudian el conjunto de interacciones que se producen en la clase entre el maestro y los alumnos acerca del sistema de escritura, del lenguaje que se escribe... 
El riesgo del enfoque "aplicacionista" consiste en que la problemática de la investigación se define en el interior (y según los fines) de la disciplina "de base", lo cual resulta ineficaz desde el punto de vista didáctico porque lleva a reducir la problemática educativa a las preocupaciones de las ciencias de referencia. Cuando se extraen consecuencias para la enseñanza a partir de saberes de otras ciencias, que no estudian la enseñanza y el aprendizaje escolar, se corre un serio riesgo de caer en el terreno de la opinión. Y el terreno de lo didáctico no es un terreno de opinión, de simple derivación de investigaciones realizadas con otros propósitos. Si pretendemos producir conocimiento didáctico, es necesario que analicemos qué es lo que sucede cuando efectivamente se desarrollan en el aula propuestas didácticas. Desde nuestro punto de vista, estas propuestas son siempre hipótesis de trabajo y estudiar su desarrollo en el aula es la única manera de entender cómo se articulan enseñanza y aprendizaje, tanto en el caso de la alfabetización como en relación con otros contenidos.

Para terminar, agrego que Schubauer-Léoni (1986) ha criticado la concepción tradicional que reserva la investigación fundamental para resolver problemas teóricos, en tanto que los problemas prácticos sólo se abordan a través de investigaciones "aplicadas". El resultado de este enfoque, señala la autora, es la ausencia de investigación fundamental sobre las prácticas sociales (como la enseñanza), es decir, sobre los problemas que revisten mayor complejidad.

MC: Hay que tener en cuenta de dónde veníamos. La didáctica específica no era una disciplina en sí misma, no tenía un objeto en particular, un objeto específico, y era un lugar precisamente de aplicación. ¿De aplicación de qué? De ciertas comprensiones y descripciones acerca de los objetos de enseñanza y, sobre todo, de las ideas y conceptualizaciones de los pequeños, a partir de las cuales se derivaba qué se tenía que enseñar, de qué manera, cuándo, etc. Lo que en realidad era una hipótesis didáctica se tomaba como verdad. La ruptura surge cuando uno se corre de ese "se debe enseñar de determinada manera" para plantearse que las disciplinas de referencia solo nos puede permitir construir hipótesis acerca de cómo enseñar. Además las aulas son diversas, entonces no es nada sencillo porque no es una prueba en el sentido experimental: si funciona de determinada manera en un aula va a funcionar de la misma manera en otra. Se trata de estudiar las situaciones pero sin intención de generalización sino de construcción de conceptos que sean útiles para poder realizar más situaciones en distintos contextos. El propósito no es construir modelos de acción sino conceptos que son herramientas teóricas para orientar la acción en distintas aulas. 
DL: Claro, no permite predecir y controlar.

MC: Permite entender y construir conceptos didácticos que tengan potencia para poder constituirse en criterios de acción en el aula.

DL: Además de orientar la acción, el propósito es también comprender las diferencias que se dan en diferentes contextos cuando se pone en práctica cierta propuesta, es también intentar explicar por qué una misma intervención del maestro puede dar lugar a respuestas muy diversas por parte de diferentes alumnos...

MC: Es un concepto didáctico fundamental: ante la misma situación se obtienen respuestas diferentes de diferentes alumnos. Lo reencontramos una y otra vez ante distintos géneros y en todas las situaciones. Avanzando sobre ello, se corrobora otro concepto didáctico central: si queremos que todos los chicos avancen debemos tener en cuenta que son necesarias intervenciones diferentes para niños en diferentes momentos de la adquisición de la lectura. "Enseñanza no es igual a aprendizaje”, en este momento, ya no es una hipótesis o una postura opinable, es una comprobación, se enseña lo mismo a un grupo de chicos y distintos niños aprenden distintas cosas. De este modo, lo que va aportando la investigación y otros modos de construcción de conceptos didácticos son herramientas para dar clase, más allá de la clase que se está investigado.

DL: También es una herramienta para planificar y para evaluar las clases.

MC: Sí. Y señalemos que también es distinta la manera de entender la relación entre investigación y docencia.

DL: Claro, tampoco hay una relación aplicacionista entre investigación didáctica y enseñanza. El maestro reelabora el conocimiento didáctico y lo adecua a su estilo de trabajo, a su grupo concreto, a su contexto. Lo que quiero decir es que tampoco creemos que los resultados de la investigación didáctica sean aplicables directamente en el aula: los docentes se apropiarán de aquellos resultados de investigación que les ayuden a resolver problemas didácticos observables para ellos, que sean reutilizables en las condiciones de su lugar de trabajo, cuya 
puesta en práctica parezca factible en el marco de las exigencias múltiples que se plantean en un aula.

TC: Actualmente se plantean perspectivas opuestas sobre la enseñanza. Algunos sostienen que la lectura y la escritura son prácticas y que por lo tanto se aprenden practicando, es decir, leyendo y escribiendo mucho, otros mantienen que esos procesos deben ser conducidos explícitamente desde la enseñanza y por último están quienes proponen un enfoque integrado o equilibrado. ¿En qué lugar ubicarían su trabajo didáctico?

MC: Hay una tendencia a pensar que hay solamente dos maneras de enseñar: una implícita y otra explícita, una indirecta y otra directa o hasta una por inmersión y otra por mostración. En realidad, nuestra concepción no corresponde a ninguna de esas categorías opuestas, sobre todo si por implícita, indirecta o por inmersión se entiende ausencia de enseñanza sistemática. No consideramos que nuestra propuesta de enseñanza sea implícita por el hecho de que no enseñemos "directamente" las relaciones fonema-grafema, por mencionar uno de los puntos de controversia. Porque tampoco es que esas relaciones no se abordan de ningún modo. Lo hacemos cuando resulta asimilable para los chicos y, mientras tanto, desplegamos otras intervenciones para lograr que esto suceda. Cuando ya pueden producir escrituras distintas para palabras o enunciados distintos, empezamos a intervenir para que interpreten las partes de la palabra y establezcan relaciones entre la interpretación del todo y la de las partes, para que vayan tomando en cuenta que las diferencias entre las escrituras se vinculan con la pauta sonora del habla. Para pensar en estas intervenciones fueron cruciales los trabajos de Sofía Vernon porque mostraron el proceso de construcción de la correspondencia sonora en la transición entre los períodos pre-silábico y silábico, así como el rol de la escritura en ese proceso. Esto no sucede con todos al mismo tiempo y hay muchos que ingresan a primero más avanzados, vinculando ya las partes de lo escrito con partes de lo oral. Para los que aún no lo hacen, que pueden estar en nivel inicial o en primero, nuestra propuesta contempla una serie de situaciones y de intervenciones específicas orientadas a generar esta reflexión, que se realiza de manera sostenida hasta que todos puedan establecer relaciones entre partes de lo oral y de lo escrito. Al mismo tiempo, la condición para que esto suceda es que todos los chicos estén inmersos en prácticas de escritura genuinas en todo momento en el aula, cualquiera sea su nivel. Pero la inmersión no es suficiente, es apenas una condición. Antes de eso, tampoco nos quedamos con los brazos cruzados esperando que produzcan escrituras 
diferentes para enunciados diferentes. Algunos niños muy pequeños o que han tenido escasísimo contacto con la escritura empiezan haciendo garabatos que, desde ellos, "dicen" algo. Con garabatos no se pueden hacer diferenciaciones; para producir escrituras diferenciadas en necesario tener un repertorio de marcas gráficas que algunos chicos no tienen en el inicio. Entonces, la intervención con ellos apunta a que adquieran variedad y cantidad de marcas (letras), las llamen como las llamen y las usen como las usen (aunque el docente siempre lo haga convencionalmente). Con ellos no vamos a empezar por reflexionar sobre las relaciones entre unidades de lengua oral y escrita porque esto no tendría sentido para ellos.

Es lo que Delia menciona cuando dice "tomarse en serio las conceptualizaciones de los chicos". Algunas intervenciones son comunes a todos los chicos pero otras son específicas para niños con posibilidades diferentes, esta es una diferencia importante con otras propuestas. Con ellos hacemos otro trabajo que tiene como marco la inmersión en la lengua escrita, igual que con todos, pero donde se abren momentos para analizar cómo escriben los otros (con "marcas" que son muchas pero no infinitas ni cualesquiera), para "escribir" con letras móviles que son muy distintas a las propias marcas, para copiar con sentido usando o intentando usar las marcas convencionales aunque se cometan errores, para "leer" enunciados que -adulto mediante- empiezan a tener partes comunes y diferentes a otros enunciados aunque en este momento -para el niño- no se correspondan con partes de la oralidad. Sería muy extenso explicar aquí todos los tipos de escrituras que pueden transitar desde los garabatos hasta la escritura diferenciada y desde los primeros intentos de poner en correspondencia lo oral y lo escrito hasta la escritura convencional, pero desde el punto de vista de la enseñanza siempre es el mismo criterio: situaciones de escritura con sentido con intervenciones comunes a todos los chicos e intervenciones específicas para niños con distintas posibilidades de escritura. En todo momento, lo que hacemos es sostener la reflexión sistemáticamente sobre las unidades de lo escrito y sus relaciones con las unidades de lo oral, aun antes de que los chicos puedan acceder a la lectura y la escritura convencional.

TC: ¿Eso sería algo que no se comparte entre perspectivas?

MC: Estamos hablando de propuestas didácticas, no de las aulas efectivas ni de teorías psicolingüísticas, aunque cada propuesta se referencie claramente en una teoría. Analizando exhaustivamente las propuestas, en este punto el panorama es dicotómico pero eso no supone que no haya ningún punto de coincidencia. 
TC: A ver, ¿en qué es dicotómico y en que coincide?

MC: Prefiero empezar por las coincidencias, que en las últimas décadas son mayores que antes. Es controvertido y difícil de asir porque muchas veces se coincide en la acción en el aula por razones teóricas diferentes.

TC: ¿Un ejemplo?

MC: La centralidad de la lectura del docente. Más o menos desde la década de 1960, se sabe que los chicos que vienen de ambientes donde se lee y se les lee mucho aprenden mejor. Por eso el Whole Language, desde el inicio, incluyó la lectura del adulto como una actividad central dentro del aula, no solo lectura de cuentos sino un menú de situaciones de lectura con sentido como lectura de recetas de cocina, de noticias, de instrucciones de juego y de toda situación de lectura con sentido que se pudiera compartir con los niños. Nosotros heredamos esas situaciones y sostuvimos, como ellos, que se empieza a leer a través del adulto. Avanzamos, porque también precisamos condiciones y tipos de intervenciones generales y específicas para distintos géneros $\mathrm{y}$, sobre todo, porque nos alejamos de la idea de que solo hace falta leer y escribir para aprender a leer y a escribir. Ahí introdujimos el concepto de reflexión sobre el lenguaje que no es lo mismo que conciencia porque no pensamos en un estado que cambia de un momento para otro sino en un proceso de reflexión. Pero, volviendo a las coincidencias, aunque distintas perspectivas no estemos de acuerdo en por qué, para qué y cómo leer en voz alta en el aula, todos sostenemos actualmente que es central que los chicos se sumerjan en lecturas, a través de la voz del docente, desde el primer día de clases y con mucha frecuencia.

TC: ¿Para aprender a leer?

MC: Para aprender los sentidos de las prácticas de lectura, que no son obvios para todos los chicos, y para aprender algo que es fundamental cuando los niños intentan leer por sí mismos: el lenguaje escrito. Por cierto que gran parte de las intervenciones del adulto conducen a hacer reparar en ese lenguaje escrito, los chicos no lo tienen especialmente en cuenta, están en general más centrados en la historia o en el contenido que en el lenguaje que se usa. Reparar en la forma del lenguaje es una práctica propia de cultura escrita que para la mayoría de los 
chicos se enseña en la escuela y requiere de conceptos específicos que se aprenden en la escuela junto a un léxico específico.

MA: ¿Qué sería el lenguaje escrito y por qué es fundamental?

MC: El lenguaje escrito, concepto que debemos a Claire Blanche-Benveniste y a Ana Teberosky, es la forma que adquiere el lenguaje en los distintos géneros y que es diferente al de la lengua oral coloquial. En lengua oral decimos, "tienes/tenés que poner unas tres cucharadas de harina para que se espese" y en lengua escrita es más probable encontrar "agregue la harina y revuelva hasta espesar", ya que en otra parte del texto se explicita que son tres cucharadas. Es fundamental que los chicos estén familiarizados con este tipo de formulaciones de lengua escrita no solo porque es la forma del lenguaje para escribir sino también porque al acercarse a los textos e intentar interpretar qué dice en la escritura, lo que tienen que anticipar son formulaciones de lengua escrita. Eso les permite hacer anticipaciones “posibles” y buscar índices para corroborar, refutar o ajustar las anticipaciones. Es algo que no todas las propuestas comparten.

MA: ¿Por qué?

MC: Porque muchas propuestas -en realidad, la mayoría, incluidas las llamadas "equilibradas"- sostienen que los chicos tienen que aprender a decodificar palabras con velocidad y precisión ya que de lo contrario la falta de "habilidad para el reconocimiento de palabras" obstaculizaría la comprensión de los textos. Esas palabras se extraen o no de textos que han sido leídos, pero en algún momento se trabaja con listas o grupos de palabras graduadas por distintos criterios, incluidas pseudopalabras. Cuando estoy frente a una lista de palabras sin contexto y sin campo semántico que las unifique, anticipar qué puede decir resulta muy difícil. Estoy solo frente a las letras y, si aún no sé leer convencionalmente, en lo único que me puedo apoyar es en la decodificación.

MA: Por eso para ustedes leer no es decodificar.

MC: No, claro que no lo es. Pero eso no quiere decir que la lectura se reduzca a anticipar formas de lenguaje escrito. Hemos sistematizado cuidadosamente las situaciones y las 
intervenciones para enseñar a los niños a leer por sí mismos. Situaciones que se caracterizan por el mayor o menor conocimiento de la forma y el contenido del texto para hacer anticipaciones e intervenciones del docente que promueven que los chicos consideren "qué puede decir", "dónde puede decir" y "cómo puede decir" si una escritura empieza, termina o contiene tales letras que pueden conocer por su nombre, su sonido o la pertenencia a otros nombres de personas $\mathrm{u}$ objetos que identifican aunque aún no puedan leer en el sentido convencional del término. Es un tipo de situaciones sobre el que hemos trabajado muchísimo. Una de las últimas sistematizaciones la hicieron Ana Kaufman y Delia Lerner para un programa de formación de docentes. Por eso, por más que no enseñamos a identificar palabras aisladas a través de la puesta en correspondencia de letras con sonidos, no nos cabe pensar que nuestra forma de enseñar es implícita si por ello se entiende librada a las posibilidades del alumno, solo por inmersión.

TC: Mencionaste que los enfoques equilibrados comparten la idea de enseñar a decodificar ¿Y entonces por qué se llaman equilibrados?

MC: En mi opinión -puedo estar equivocada, lo afirmo en función de sostener la lectura y el análisis bastante sistemático de distintas propuestas latinoamericanas-, se debe a que son enfoques que antes partían de palabras que se analizaban hasta la relación fonema-grafema y el lenguaje escrito como tal no estaba presente sino mucho más adelante, cuando ya se habían enseñado todas las letras, o más aún (porque el genuino lenguaje escrito no son los textos exclusivamente escolares). A partir de los estudios de los 60 y el movimiento del Whole Language, se fue advirtiendo que presentar la cultura escrita en el aula es indispensable, especialmente en sectores populares. Entonces plantean dos ejes de trabajo, uno que mantiene las actividades de codificación y decodificación y otro que introduce la cultura escrita. Son "equilibrados" en relación a propuestas que solo tienen en cuenta las unidades menores del sistema. Pero nosotros no nos consideramos a nosotros mismos "desequilibrados" por tener una concepción distinta de la lectura y la escritura. Hoy nadie plantearía que no es necesaria la cultura escrita dentro del aula.

TC: ¿Hay alguna otra diferencia? 
MC: Ante todo, no quiero olvidar otra coincidencia, aunque las razones de la misma, como dije, no resida en coincidencias teóricas. En las propuestas actuales de las otras posturas hay mucha más presencia de escritura de lo que había originalmente. Para nosotros la escritura siempre fue el corazón de la propuesta, no sólo porque consideramos imprescindible abrir un espacio para que los chicos escriban según sus propias conceptualizaciones acerca de ese sistema de representación sino también porque provee de un material discontinuo y analizable que la sola oralidad no permite. Por eso nunca proponemos situaciones solo sobre el análisis de la emisión oral para después "aplicar" a lo escrito. En todas nuestras situaciones está y estuvo presente la escritura desde un inicio. En la perspectiva de Conciencia Fonológica y en los enfoques equilibrados esto no es así, hay un eje de trabajo que es análisis de la oralidad sin escritura o, al menos, lo era hasta hace algunos años. Ahora, en esas perspectivas ese eje se puede mantener pero además hay análisis de la emisión oral en presencia de escritura. Aunque para otras perspectivas la facilitación provenga de la "exposición al escrito" y para nosotros se trate de un proceso reconstructivo de la lengua oral vinculado con los esfuerzos de los niños por comprender la escritura; nos alegra su presencia en el aula porque, cuando no está presente, los niños se ven obligados a analizar una cadena sonora sin apoyatura alguna. Es en la concepción misma de la relación entre oralidad y escritura donde residen las diferencias. La escritura no es un reflejo de lo oral ni se pueden escuchar en la oralidad unidades cuya existencia depende de la escritura. Son perspectivas epistemológicas radicalmente distintas que no solo se manifiestan en la concepción del objeto. También el sujeto se concibe de manera diferente. Cuando un chico escribe "AOEA" para "mayonesa", no pensamos que no escucha las consonantes sino que su escritura está orientada por la idea de que a cada sílaba de la oralidad le corresponde una letra en la escritura. Investigaciones recientes de Molinari y Ferreiro muestran niños que escriben alternativamente "AOEA" y "MYNS", en el curso de una misma entrevista, lo que sugiere que ellos pueden ya establecer cuáles son todas -o casi todas- las letras que componen esa palabra escrita pero su conceptualización de la escritura sigue siendo silábica. Más allá de que con otras metodologías de indagación y desde otros marcos interpretativos se haya cuestionado la idea de hipótesis infantiles, específicamente la hipótesis silábica, nuestra concepción del error es diferente y eso se manifiesta fuertemente en las propuestas didácticas. En nuestra perspectiva se anima a los chicos a escribir de acuerdo a sus hipótesis y se los ayuda a avanzar desde donde están pero sin pretender que pasen bruscamente a la escritura convencional. No pensamos que haya que evitar las escrituras no convencionales sino todo lo contrario, porque en la medida en que experimenten más con la 
escritura van a tener más oportunidades de enfrentar conflictos que los impulsen a avanzar. Hay trabajos recientes, como el de Regina Scarpa, que muestran que los chicos avanzan más cuando se les permite escribir de maneras no convencionales. Se podría decir mucho acerca de por qué distintas perspectivas conciben la evolución de la escritura de manera diferente, pero sería una entrevista demasiado extensa.

MA: Pensando ahora sobre otras cuestiones, ¿qué condiciones políticas, institucionales, de formación y trabajo docente deben asegurarse para abordar la alfabetización desde esta perspectiva?

DL: Hemos trabajado en condiciones muy diversas en diferentes países, al menos en relación con las políticas públicas y con la formación de los docentes. Por eso, en lugar de centrarme en cuáles serían las condiciones ideales que habría que asegurar, preferiría pensar en cómo se fue concretando nuestro proyecto de enseñanza en las condiciones reales en las que trabajamos, así como en las modificaciones que ha sido posible promover.

Comienzo por las condiciones institucionales, que son quizá las más constantes en diferentes contextos. La graduación es un rasgo fuerte de la escuela y es sin duda una condición adversa para un proyecto de alfabetización que se propone que todos los niños se formen como lectores y escritores.

MA: ¿En qué sentido sería una condición adversa?

DL: El dispositivo escolar graduado encarna el supuesto de que, cuando se enseña cierto contenido, todos los alumnos aprenden lo mismo al mismo tiempo, es la vocación homogeneizadora de la escuela moderna, es lo que Flavia Terigi ha llamado la monocronía de los aprendizajes. Desde nuestra perspectiva didáctica, y en particular en relación con la alfabetización inicial, saber que los niños comienzan la escolaridad primaria con diferentes conceptualizaciones acerca del sistema de escritura y que no todos han tenido las mismas oportunidades de participar en diferentes prácticas de lectura y escritura exige prever la diversidad y tomarla en consideración al interactuar con los alumnos.

Como ha señalado Roland Goigoux, enseñar exige "conducir la trayectoria intelectual de la clase en su conjunto sin perder de vista la trayectoria de cada sujeto singular". No es fácil. Reconocer la diversidad y proponerse que todos aprendan -sabiendo que no todos 
aprenderán lo mismo- es un desafío para la escuela y también para el saber pedagógico y didáctico.

MA: ¿Y cómo se resuelve eso?

DL: Por el momento no es posible resolverlo integralmente. No obstante, muchas de nuestras producciones de los últimos años se centran en aportar conocimientos sobre las intervenciones de los docentes que pueden favorecer el aprendizaje de todos en el marco de situaciones de lectura y escritura. Una propiedad general de estas intervenciones es la de engarzarse con el estado de conocimiento de los chicos, con sus hipótesis, con sus interpretaciones. Tal como acaba de mostrarlo Mirta en relación con la reconstrucción del sistema de escritura, se trata de intervenir desde dentro de lo que ellos están pensando, de plantearles problemas que los inciten a desarrollar o a cuestionar sus ideas y que, por eso mismo, los ayuden a avanzar.

En muchos casos, es necesario producir cambios en la organización escolar. Para que los docentes puedan efectivamente intensificar sus intervenciones y articularlas con los diversos estados de conocimiento de los alumnos, hay que generar modalidades de agrupamiento alternativas a las usuales. Una modalidad que ha resultado productiva consiste en hacer reagrupamientos provisorios que reúnen -por ejemplo, una o dos veces por semana durante cierto período- a niños de diferentes grupos de un mismo grado cuyas conceptualizaciones acerca del sistema de escritura sean cercanas. Los maestros de un grado tres maestros de primero, por ejemplo- planifican juntos las actividades a proponer y cada uno de ellos se hace cargo de coordinar uno de los nuevos reagrupamientos. Cuando es posible, el personal directivo y algún otro docente de la escuela participan también en estas actividades. Pudimos plantear gran parte de estas formas de reagrupamiento, que fuimos sistematizando a partir de experiencias en distintas escuelas, en un documento sobre evaluación (Kaufman, Lerner, Castedo, Torres, 2015). Modificaciones organizativas como la que acabo de describir permiten flexibilizar la organización de los grupos, lo que contribuye a ampliar las posibilidades de interacción cognitiva con diferentes compañeros y también con diferentes maestros. Es una cuestión en la que han trabajado muchas colegas, entre las cuales quisiera destacar a Myriam Nemirovsky. Por otra parte, estas modificaciones organizativas hacen posible atenuar otra condición adversa del dispositivo escolar: la soledad del trabajo docente. 
MA: ¿Consideras que la soledad del trabajo docente es una condición a revisar por los sistemas educativos?

DL: Sin duda. Poner en común miradas de diferentes maestros sobre el trayecto de los niños con los que todos han tenido oportunidad de trabajar, pensar juntos en situaciones didácticas que pueden contribuir a su progreso, compartir el análisis del desarrollo de las actividades planteadas y re-planificar, articular el trabajo en el aula entre maestros de años consecutivos...

Todo esto hace posible -hemos podido constatarlo en diferentes ámbitos institucionales y en diversas instancias de formación- no sólo construir propuestas más productivas para el aprendizaje sino también problematizar la enseñanza, enriquecer los conocimientos didácticos de los docentes e ir tejiendo una trama de colaboración que permite a cada maestro sentirse respaldado al asumir el desafío de lograr que todos sus alumnos logren apropiarse del sistema de escritura y avancen como lectores y escritores (Lerner, 2012).

TC: ¿Crees que esas experiencias podrían generalizarse?

DL: En ciertas circunstancias, cuando nuestros proyectos de enseñanza pueden enmarcarse en políticas públicas orientadas a democratizar la educación, se producen modificaciones de mayor alcance en el dispositivo escolar. Es lo que ha sucedido en diferentes países latinoamericanos que produjeron una primera ruptura con la escuela graduada al instituir los dos primeros años de escolaridad como una unidad sin promoción interna, lo que hace posible que muchos niños se alfabeticen mejor $\mathrm{y}$, en consecuencia, se evita o se disminuye considerablemente la repitencia. Fue pionero en este sentido el Ciclo Básico instituido por la Secretaría de Educación del Estado de Sao Paulo (Brasil) en la década de 1980 y, entre las modificaciones posteriores, menciono sólo una reciente: la Unidad Pedagógica establecida en Argentina a partir de la Resolución 174 del Consejo Federal de Educación (2012), dirigida a asegurar la continuidad de las trayectorias escolares de todos los niños.

TC: Una última pregunta: a lo largo del trabajo que han desarrollado en formación docente, ¿fue posible explicitar condiciones necesarias para que los docentes participantes puedan 
apropiarse de conceptos o estrategias de enseñanza que contribuyan a la formación de los alumnos como lectores y escritores?

DL: Hemos trabajado sobre todo en formación docente continua. Después de muchos años de experiencia -sobre todo en el marco de programas que, por articularse con una política pública, abarcaron una cantidad apreciable de docentes e implicaron la constitución de equipos de formadores- elaboramos algunas conclusiones. Sólo voy a mencionar algunas de las que consideramos fundamentales: los procesos formativos que logran producir algún impacto en las prácticas son aquellos que se desarrollan durante períodos prolongados, que ponen en primer plano el análisis de las clases e incluyen la planificación compartida y el acompañamiento en el aula. Estas estrategias se articulan con otras -la discusión de material bibliográfico, el análisis de producciones infantiles, la escritura profesional, etc.- y es esta articulación la que va haciendo posible que los docentes construyan progresivamente herramientas teóricas productivas para interpretar la práctica y para re-orientarla considerando los aprendizajes de sus alumnos, los problemas cognitivos que enfrentan y las intervenciones que contribuyen a resolverlos. Hay diversas publicaciones sobre esto, por eso me limitaré a subrayar que, en los últimos años, estamos enfatizando fuertemente el papel de los maestros como productores de conocimiento y el trabajo compartido entre docentes tanto dentro como fuera del aula.

Nos parece imprescindible profundizar en el estudio de los procesos de formación y estamos avanzando en ese sentido, gracias a la documentación producida por algunos de los programas realizados y también gracias a la elaboración de tesis centradas en diferentes facetas de ciertos procesos formativos. Hay mucho camino por recorrer en este sentido. También quedan problemas importantes por resolver en el campo de la alfabetización inicial, por eso la investigación didáctica continúa y, seguramente, continuará.

\section{Referencias}

Ferreiro, E. (2009). La desestabilización de las escrituras silábicas: alternancias y desorden con pertinencia. Revista Lectura y Vida: Revista latinoamericana de lectura, 30(2), 613.

Kaufman, A., Lerner, D., y Castedo, M. (2015). Leer y aprender a leer. Especialización docente de nivel superior Alfabetización en la Unidad Pedagógica. Buenos Aires: Ministerio de Educación de la Nación. 
Kaufman, A., Lerner, D., Castedo, M. y Torres, M. (2015). Seminario acerca de la evaluación. Especialización docente de nivel superior Alfabetización en la Unidad Pedagógica. Buenos Aires: Ministerio de Educación de la Nación.

Goigoux, R. (2007). Un modèle d'analyse de l'activité des enseignants. Éducation et Didactique, 1(3), 47-69.

Lerner, D. (2012) Como o trabalho compartilhado entre os docentes favorece o aprendizado dos alunos. Nova Escola. Janeiro, 2012. Recuperado de https://novaescola.org.br/conteudo/546/como-o-trabalho-compartilhado-entre-osdocentes-favorece-o-aprendizado-dos-alunos

Molinari, C., y Ferreiro, E. (2007). Identidades y diferencias en la escritura en papel y en computadora en las primera etapas del proceso de alfabetización. Revista Lectura y Vida: Revista latinoamericana de lectura, 28 (4), 18-30.

Nemirovsky, M. (1999). Interacción entre niños de diferentes grupos escolares. En: Sobre la enseñanza del lenguaje escrito... y temas aledaños (pp. 71-85). México: Paidós.

Scarpa, R. (2014). O conhecimento de pré-escolares sobre a escrita: impactos de propostas didáticas diferentes em regiões vulneráveis (tesis de doctorado). Brasil: Universidade de São Paulo- Faculdade de Educação.

Schubauer-Léoni, M. (1986). El desarrollo cognitivo de los niños en la escuela primaria. La psicología del aprendizaje en las diferentes situaciones pedagógicas. Revista de Educación, 279, 103-120.

Terigi, Flavia (2010): El saber pedagógico ante la crisis de la monocronía. En: Frigerio, G. y Diker, G (eds.), Educar: saberes alterados (pp. 99-110). Paraná: Del Estante.

Vernon, S. (1997). La relación entre la conciencia fonológica y los niveles de conceptualización de la escritura (tesis de doctorado). México. DIE-CINVESTAVIPN.

Vernon, S. (1991). El proceso de construcción de la correspondencia sonora en la escritura (en la transición entre los períodos presilábico y el silábico) (tesis de maestría). México: DIE-CINVESTAV-IPN.

\footnotetext{
${ }^{\mathrm{i}}$ Delia Lerner se refiere al Diseño Curricular del área de Prácticas del Lenguaje de la Provincia de Buenos Aires, Argentina.
} 


\section{Información sobre las autoras:}

Marta Alegría es estudiante en el Doctorado en Ciencias de la Educación, de la Universidad Nacional de La Plata, Argentina. Su investigación estudia el desarrollo de situaciones didácticas de edición de textos con niños de educación primaria, bajo la dirección de Mirta Castedo (UNLP) y Celia Díaz (UNAM). En 2014 obtuvo un Magíster en Escritura y Alfabetización en la Universidad Nacional de La Plata. Actualmente es docente de Didáctica del Lenguaje y Diversidad en la carrera de Educación Especial de la Universidad Alberto Hurtado, Chile.

Email: martaalegria@live.cl

Tatiana Cisternas es Académica e Investigadora de la Facultad de Educación de la Universidad Alberto Hurtado. Directora de la carrera de Educación Especial en esa misma universidad. Ha desarrollado diversas investigaciones vinculadas a la formación inicial y continua de docentes en Chile y en torno a las prácticas pedagógicas y saberes en distintos momentos de la vida profesional, particularmente en docentes nóveles. A nivel de pre y posgrado, imparte cursos de didáctica y atención a la diversidad, metodología de la investigación cualitativa e investigación didáctica.

Email: tatiscister@gmail.com

Para citar este artículo:

Alegría, M. y Cisternas, T. (2018). Alfabetización inicial desde una perspectiva constructivista psicogenética. Una entrevista con Delia Lerner y Mirta Castedo. Bellaterra Journal of Teaching \& Learning Language \& Literature, 11(2), 100-119. DOI: http://doi.org/10.5565/rev/jtl3.774

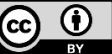

